\title{
MANCHESTER DECODER WITH HIGH ROBUSTNESS
}

\author{
Zoran T Lovreković \\ Higher Education Technical School of Professional Studies, Serbia \\ lovrekovic@vtsns.edu.rs
}

Original Scientific Paper

doi:10.5937/jouproman7-23705

\begin{abstract}
There are some diferent principles for realisation of the Decoder of the Manchester signal: -Decoder developed with the active filters, decoder based on the oscilator with codin signal controled frequency of clock, and decoder with PLL circuit, are the usual way for the realisation of that device. The suggested realisation of the decoder of Manchester signal is very robust, which is seen in the possibility, opposite to other types of Manchester decoders, to work properly even if the signal interval of the input signal vary up to $\pm 25 \%$, and in a very simplified realisation.
\end{abstract}

Key words: Manchester decoder, robustness, Manchester coded signal

\section{Introduction}

The advantages of Manchester coding over unipolar binary non-return to zero signal NRZ are[1], [2]:

- easy clock extraction,

- constant value of DC component of signal,

- there is no variation in the optical power of the laser, if signal is transmitted optically.

The format of the Manchester code is such that there are no long intervals of logical 1 or 0 , which cause the aforementioned variations in the optical power of the source. Due to the fact that it does not require long continuous radiation, the life of the laser diode increases. On the other hand, the Manchester coded signal requires double width of the bandwidth of the transmission fiber in relation to the corresponding NRZ signal[3].

The Manchester encoder itself is a very simple and one example of such an encoder is given in Figure 1.

Observing the NRZ waveforms and the corresponding Manchester signal, it can be seen that information on the value of the decoded signal at a given clock interval is given by the Manchester coded signal transition at exactly half of that clock interval. Possible transitions of the Manchester coded signal at the ends of the clock intervals do not carry any information about the value of the decoded signal. Thus, it is necessary to extract useful transitions of the encoded signal located at $(2 \mathrm{k}-1) *$ Ts $/ 2, \mathrm{k}=1,2, \ldots \ldots . \mathrm{It}$ is noted that these transitions are exactly at a distance from $\mathrm{Ts}$, which makes it easy to extract clock.

There are several different principles on which is possible to base the realization of a Manchester signal decoder[1]:

- Decoder based on active filters

- An oscillator based decoder with a clock frequency controlled by a coded signal

- Decoder with PLL (Phase Locked Loop) circuit 


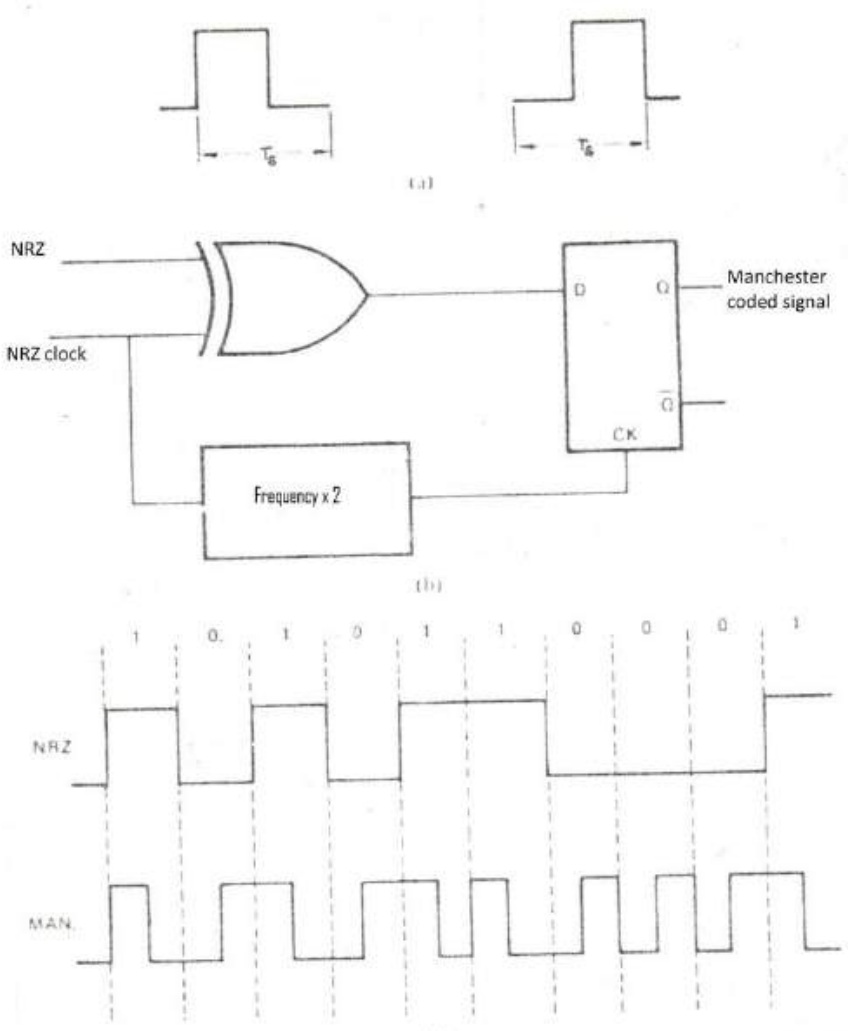

Fig.1. Manchester coder

\section{Manchester Decoder with high} resistance to variations in signaling interval

The PLL circuit based decoder allows for some variation in the frequency of signaling speeds (about $\pm 12.5 \%$ ). The decoder shown in the Figure 2 allows the signaling interval to be changed by up to \pm $25 \%$ and the decoder still remains safe. In addition, the implementation of this decoder is very simple. The principle of operation of this decoder is easiest to understand by observing the scheme and the waveforms at the characteristic points given in Figure 3.

The Q outputs of the monostable multivibrators are in a stable state at logic value 1 , ensuring that the inputs to AND gates that are comming from the outputs of the monostable multivibrators are at the logical value 1 . 


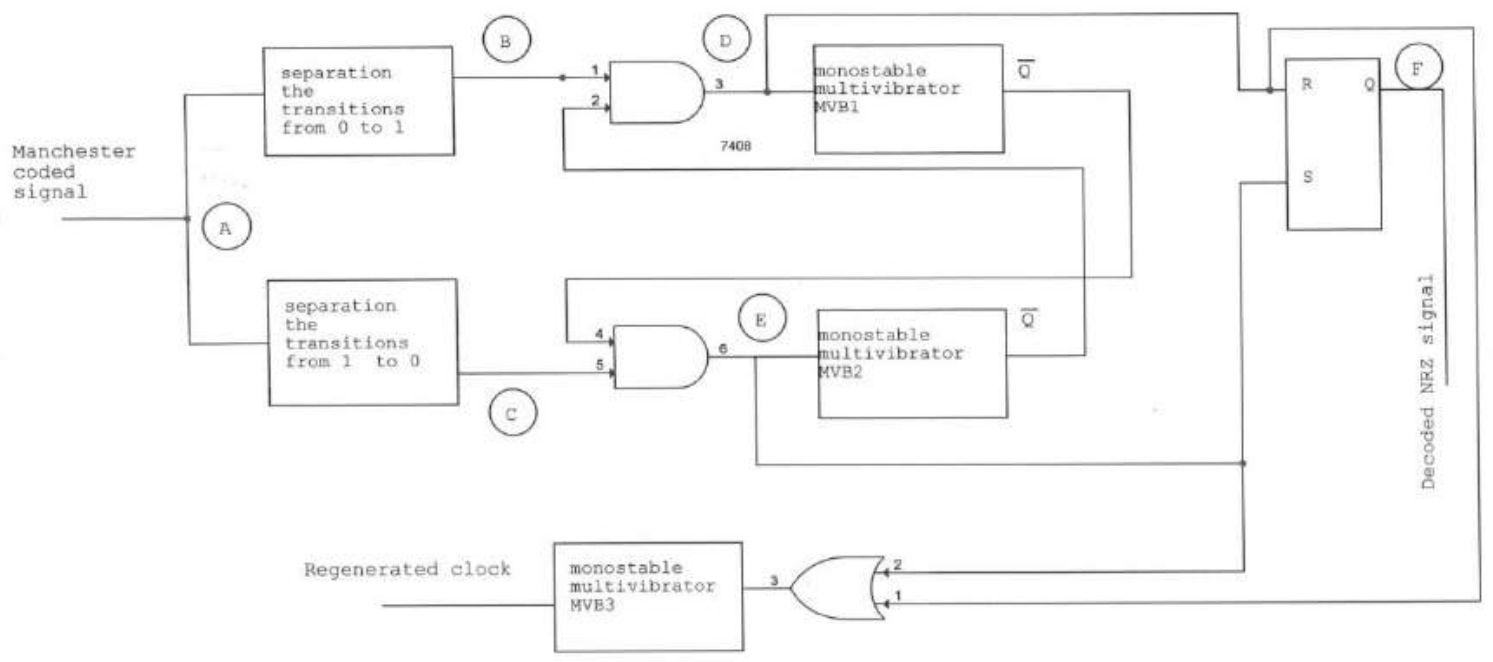

Fig. 2 Manchester decoder with high robustness

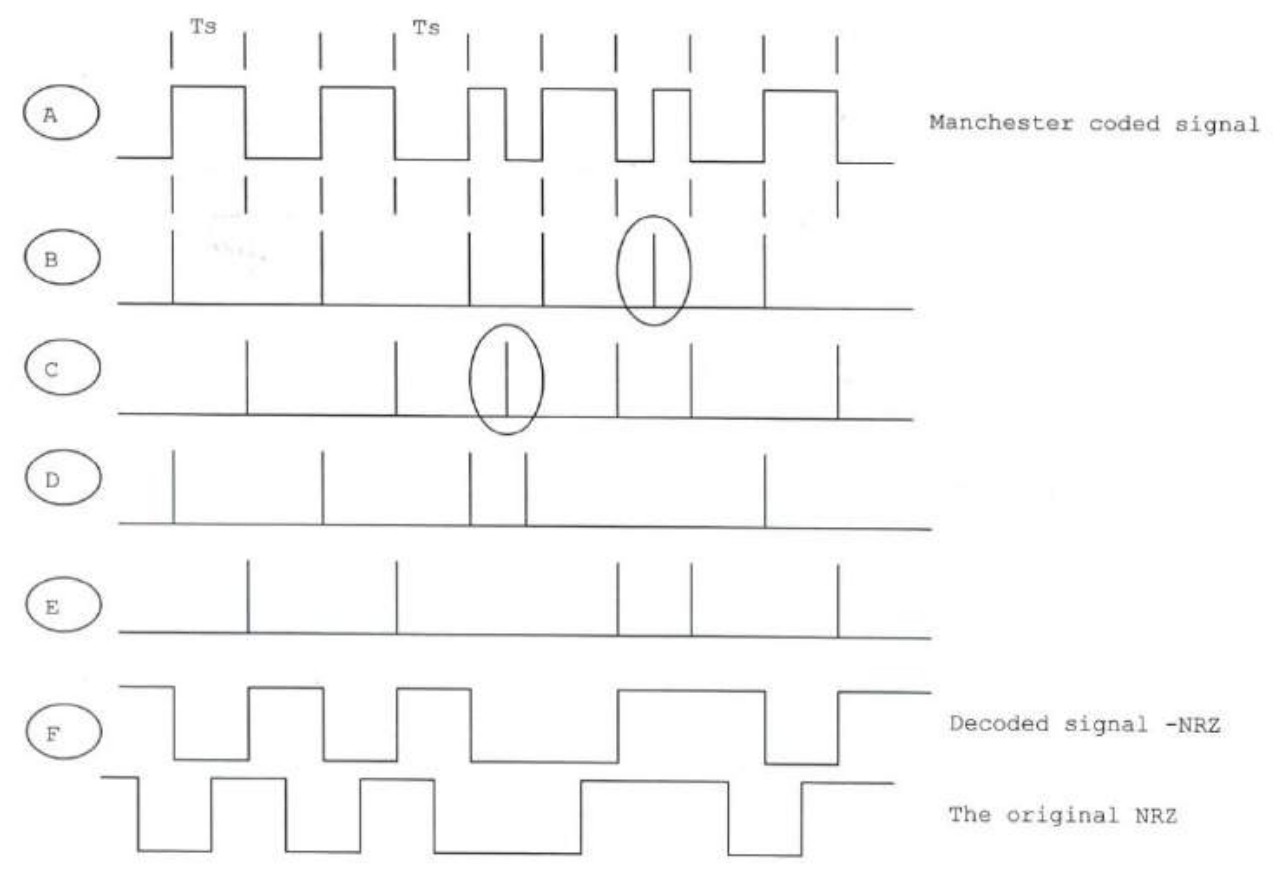

Fig. 3 Waveforms at the characteristic points of Manchester decoder given at Figure 2.

Upon arrival of the input Manchester coded signal, a positive impulse corresponding to the ascending edge of the signal will be separated and will pass through the corresponding AND gate, thus triggering the monostable multivibrator 1 . 
During Tmvb, multivibrator is in the excited, unstable state, and its output Q will be at the logic 0 value, so the second AND gate will be impassable for the impulse obtained by separating the descending edge of the input signal during Tmvb. Simultaneously with the release of monostable multivibrator 1 , a reset of the output flip-flop which is initially set will be performed, which corresponds to the signal decoding process. If the condition is satisfied that the

\section{$\mathrm{Ts} / 2<\mathrm{Tmvb}<\mathrm{Ts}$}

an impulse pass that indicating a Manchester encoded signal transition that does not carry information about the value of the decoded signal will be prevented. The impulse corresponding to the descending edge of the encoded signal will set the flip-flop, thus ensuring the continuation of the correct decoding process, provided that it represents a transition at the half and not at the beginning or end of the clock interval, which is provided by a monostable mvb with a Tmvb period as indicated. This impulse will also excite the monostable multivibrator 2 , which will ensure that the next impulse which is corresponding to the transition of the coded signal from 0 to 1 passes the through AND gate, but only if this transition carries information about the value of the decoded signal.

In Figure 3, at the characteristic signals at points $\mathrm{B}$ and $\mathrm{C}$, the impulses that do not carry information about the state of the decoded signal are rounded off, and they are eliminated as described, so that they are not present at points $\mathrm{D}$ and $\mathrm{E}$ respectively.
The formation of a clock signal is performed by a single OR gate whose output produces trigger pulses with a distance between them of exactly Ts. These impulses trigger the MVB3 monostable multivibrator, which has an excited state duration is:

$$
\mathrm{Tmvb} 3=\mathrm{Ts} / 2
$$

It is noted that the decoded signal has a delay of Ts / 2 relative to the original signal, which in the application is not a problem.

In order to be allowed a variation of the signaling interval of the input signal of \pm $25 \%$, it is necessary that the monostable state of the multivibrator Tmvb be

$$
\mathrm{Tmvb}=3 / 4 * \mathrm{Ts}
$$

If, at the moment of startup, there was an incorrect decoding, after only two bits, the decoder automatically enters the regular state.

Separation of the leading edge of the signal (transition from 0 to 1 ) is realized by the circuit of Figure 4, and the rear edges (transition from 1 to 0 ) of the circuit of Figure 5 [4] 


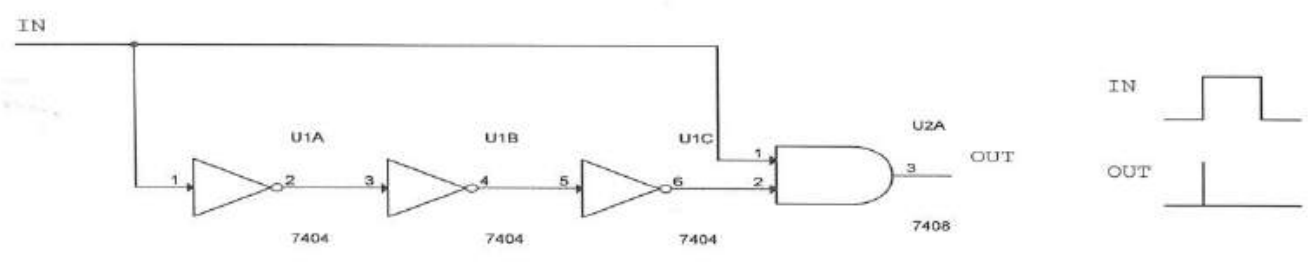

Fig. 4 Separation of the leading edge of the signal (transition from 0 to 1 )

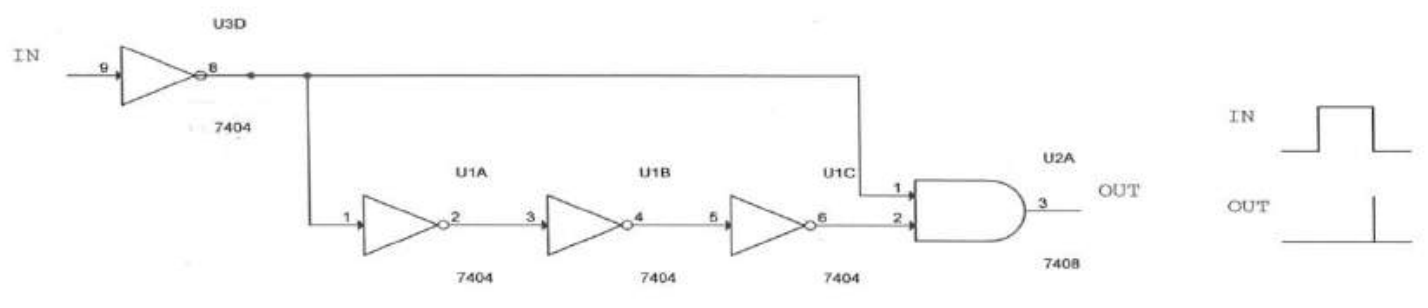

Fig. 5 Separation of the rear edge of the signal (transition from 1 to 0 )

\section{Conclusion}

The advantages of Manchester coding over NRZ in optical digital signal transmission systems are the constant value of DC component of signal, the absence of variations in the optical power of the laser, and the extended lifetime of the laser diode.

The implementation of the decoder with the PLL circuit allows for a variation of about \pm $12.5 \%$ of the signaling speed of the input signal, and realization with active filters or with an oscillator controlled by a coded signal allowing even less variation.

The designed decoder described in this paper allows for variations in signaling speed of $\pm 25 \%$. In addition, compared to other types of Manchester encoded signal decoders, it is extremely simple and easy to implement. The described decoder is extremely safe in operation, and in case of decoding error, the decoder itself enters the regular state after only two bits of the input signal.

\section{References:}

1. https://publications.waset.org/3590/pdf , Khorwat, I. A., Naas N., (2010), A New Hardware Implementation of Manchester Line Decoder, World Academy of Science, Engineering and Technology International Journal of Electronics and Communication Engineering Vol:4, No:9.

2. http://www.signalpro.biz/mandec.pdf

3. Muoi, T., V., (1983), Receiver Design for Digital Fiber Optic Transmission Systems Using Manchester (Biphase) Coding, IEEE Trans. on Communications.

4. Green, D., (1986), Modern Logic Design, Addison-Wesley Publishing Company, Wokingham, England. 